\title{
Unberechenbar? Algorithmuskulturen und ihre räumlichen Implikationen nebst einigen Bemerkungen zur geographi- schen Bildung
}

\author{
* dorsch@geo.uni-frankfurt.de, Institut für Humangeographie, Goethe-Universität Frankfurt am Main \\ **I.fuchs@em.uni-frankfurt.de, Institut für Humangeographie, Goethe-Universität Frankfurt am Main \\ ${ }^{* * *}$ kanwischer@geo.uni-frankfurt.de, Institut für Humangeographie, Goethe-Universität Frankfurt am Main \\ eingereicht am: 17.03.2021, akzeptiert am: 19.07.2021
}

Im Zuge der fortschreitenden Digitalität finden die Praktiken des alltäglichen Lebens - bewusst oder unbewusst - zunehmend in Algorithmuskulturen statt. Hierbei eröffnen sich aus räumlicher Perspektive neue fachliche Diskurse. Diese werden mittels drei verschiedener Rollen von Algorithmen in sozio-technischen Systemen aufgezeigt und auf ihre Bedeutung für raumwirksame Prozesse diskutiert. Vor diesem Hintergrund werden wir Ansätze zur Integration von Algorithmuskulturen in der geographischen Bildung vorstellen, die das mündige Agieren in einer durch Algorithmen geprägten Lebenswelt fördern.

Keywords: Digitalität, Algorithmen, Mündigkeit, geographische Bildung

\section{Unpredictable? Algorithmic cultures and their spatial implications along with some notes on geographical education}

With advancing digitality, practices of everyday life - consciously or unconsciously - are increasingly taking place in algorithmic cultures. From a spatial perspective, this initiates new professional discourses. We will illustrate these along three different functions of algorithms in socio-technical systems. Subsequently, we will discuss algorithms in terms of their significance for processes of spatial effectiveness. Finally, we will present approaches to algorithmic cultures in geographic education that promote emancipated action in a world shaped by algorithms.

Keywords: digitality, algorithms, maturity, geographical education

\section{Einführung}

Die Streaming-Plattform Spotify veröffentlichte Ende 2020 die Mitteilung, dass zukünftig Musiker*innen und Musiklabels Einfluss auf den plattforminternen Algorithmus namens „Echo Nest“ nehmen können, indem sie die Möglichkeit bekommen, einzelne Songs als besonders wichtig zu markieren. Diese Information soll auch in die Entscheidungsfindung des Algorithmus einfließen, um nutzer*innenspezifische Empfehlungen vorzuschlagen (vgl. Spotify 2020). Neben der damit einhergehenden Diskussion über Machtbeziehungen in der Musikindustrie und die Frage, wie einzelne Titel Bekanntheit erlangen, werden in der Diskussion auch die Rollen von Mensch und Maschine bei der Generierung von algorithmusbasierten Ent- scheidungen ausgehandelt. Der Erfolg von Echo Nest, der weltweit zu den erfolgreichsten Empfehlungsalgorithmen gehört, beruht auf einer Vielzahl von Informationen, die gesammelt werden, um individualisierte Musikvorschläge bereitzustellen (vgl. Spotify 2020). Dies geht so weit, dass aus den Nutzer*innendaten Ort und Zeit herausgefiltert werden, um uns an bestimmten Orten zu bestimmten Zeiten, wie z. B. auf dem Weg zur Arbeit oder im Fitnessstudio, bestimmte Musik vorzuschlagen. Damit ergibt sich eine neue Art und Weise von Musikdistribution und -konsum, die Musikproduzent*innen und -konsument*innen räumlich vollkommen voneinander entkoppelt. Dieses Fallbeispiel über die zukünftige Entwicklung von „Echo Nest“ zeigt exemplarisch, wie sich alltägliche Praktiken im Zuge der Algorithmisierung der Gesell- 
schaft verändern. Musste Musik bisher in Form von CDs oder digitalen Downloads von Hörer*innen bewusst ausgewählt, als Eigentum erworben und zum Ort des Konsums (Party, Fitnessstudio etc.) mitgebracht werden, so ist dies mittlerweile obsolet.

Was auch immer im Programmcode eines Algorithmus passiert, kann oftmals nur die Organisation nachvollziehen, die ihn entwickelt hat, und, wenn es sich um selbstlernende Algorithmen (Stichwort: Maschinelles Lernen) handelt, auch nur teilweise. Das Ergebnis ist, dass unsere Handlungen von Regeln geprägt sind, die wir nicht kennen.

Algorithmische Vorschlagssysteme ermöglichen uns eine Orientierung im und Navigation durch das Datenmeer, ohne uns die Kriterien des Entscheidungsprozesses offen zu legen. Gleichzeitig beeinflussen sie unsere Handlungen, wodurch auch unsere räumlichen Aneignungs- und Wahrnehmungsmuster beeinflusst werden. Angesichts dieser Entwicklung ist davon auszugehen, dass Algorithmen die Art und Weise der subjektiven Wahrnehmung - auch von Raum - wesentlich mitbestimmen und die Praktiken des alltäglichen Lebens formen. Dies führt wiederum zu Veränderungen des Selbstbezugs und der Weltanschauung, was zu veränderten sozialräumlichen Strukturen führt, die wiederum unser individuelles und gesellschaftliches Leben verändern. Algorithmen sind somit zugleich Teil der Welt wie auch „Weltlieferanten“.

Demnach interagieren wir in unserem Berufs- und Alltagsleben unausweichlich mit Algorithmen - ob bewusst oder unbewusst. Ob die gesellschaftlichen und räumlichen Konsequenzen dieser sozio-technischen Systeme erstrebenswert sind oder nicht, kann nur diskutiert werden, wenn wir uns darüber verständigen, welche Bereiche unseres Lebens durch welche Formen von Algorithmen geprägt werden.

Dabei eröffnen sich aus der räumlichen Perspektive neue fachliche Diskurse und damit einhergehend auch neue fachdidaktische Fragestellungen. Diese möchten wir in unserem Beitrag diskutieren. Im Fokus steht dabei die Frage, wie sogenannte Algorithmuskulturen systematisch im Kontext von räumlichen Fragestellungen im Bildungsbereich thematisiert werden können.

Hierfür werden wir zunächst einige zentrale Begrifflichkeiten im Bereich der Informations- und Kommunikationstechnologien (IKT) beleuchten, um uns dem Begriff des „Algorithmus“ anzunähern sowie die Beziehungen zu damit einhergehenden wegweisenden computertechnischen Entwicklungen $\mathrm{zu}$ verdeutlichen. Darauf aufbauend werden soziokulturelle Implikationen und deren Bedeutung für die Konstitution von Algorithmuskulturen aus sozial- und kulturwissenschaftlicher Perspektive diskutiert. Hierbei werden drei verschiedene Rollen von
Algorithmen in sozio-technischen Systemen konzeptualisiert, die für die Planung und Analyse von Unterricht genutzt werden können. Diese Rollen werden aus einer geographischen Perspektive heraus aufgegriffen und im Hinblick auf ihre Bedeutung für raumwirksame Prozesse geschärft. Das so entwickelte Schema dient abschließend dazu, Ansätze zur Integration von Algorithmuskulturen in der geographischen Bildung zu diskutieren und die in ihnen jeweils zum Ausdruck kommende Rolle von Algorithmen zu identifizieren.

\section{Vom Algorithmus zu Data Science}

In den folgenden Abschnitten werden zentrale Treiber der beschriebenen Veränderungen im Hinblick auf die algorithmische Verarbeitung digitaler Daten beleuchtet. Vor dem Hintergrund der enormen Vielfalt an technologischen Entwicklungen innerhalb der letzten Jahrzehnte, kann dieses Kapitel nur ein Schlaglicht auf einzelne ausgewählte Phänomene darstellen, welche die Entwicklung von Algorithmuskulturen beschleunigen. Gleichwohl möchten wir hiermit einerseits unsere wichtigsten Bezugsquellen offenlegen und andererseits die aus unserer Sicht grundlegenden Entwicklungen skizzieren. Die Auswahl der Phänomene stützt sich maßgeblich auf die Ausführungen von Stalder (2016) und Beiträge aus dem Sammelband „Algorithmuskulturen“"von Seyfert und Roberge (2017), welche durch Literatur aus dem Bereich der Computerwissenschaft ergänzt wurde. $\mathrm{Zu}$ Beginn werden wir uns zunächst dem Begriff „Algorithmus“ annähern.

\subsection{Algorithmen}

Zunächst ist festzustellen, dass der Begriff „Algorithmus", wie er in der sozialwissenschaftlichen Literatur in seiner ganzen Komplexität thematisiert wird, die ursprüngliche Bedeutung des Wortes aus mathematischinformatischer Sicht tendenziell stark überzeichnet. So ist ein Algorithmus als einfache Wenn-DannVerknüpfung nicht gleichzusetzen mit komplexen Architekturen der digitalen Informationsverarbeitung, wie sie im Feld der aktuellen Softwareentwicklung Anwendung finden. Systeme, wie der eingangs skizzierte Distributions-Mechanismus von Spotify stellen eine Verknüpfung oder Aneinanderreihung einer Vielzahl einzelner Algorithmen dar, welche anhand einer festgeschriebenen Logik miteinander interagieren und sich somit gegenseitig beeinflussen. Zur besseren Einordnung möchten wir den Begriff, ausgehend von einem abstrakten Verständnis hin zu einer greifbaren, gegenständlichen Vorstellung digitaler Algorithmusassemblagen, nachvollziehen. 
Algorithmen können grob definiert werden als ein festes Schema von Regeln, die die Handlungen von Maschinen oder Menschen steuern. Nicht selten werden deshalb Kochrezepte oder Bauanleitungen für Möbel als Beispiel für Algorithmen angeführt. Das Ziel der Ausführung von Algorithmen ist dabei die Lösung vordefinierter Probleme. Robert Kowalski konzeptualisiert den Algorithmus aus der Summe der Komponenten „Logik“ und „Kontrolle“. „Logik“ umfasst dabei das Wissen, das über ein Problem vorhanden ist und „Kontrolle" beschreibt die Art und Weise, wie dieses Wissen eingesetzt wird, um dieses Problem zu lösen (vgl. Kowalski 1979: 425). Die Kontrollkomponente des Algorithmus kann dabei durch drei zentrale Merkmale charakterisiert werden (Stalder 2016: 167 f.):

1. Die Schritte müssen einzeln und in ihrer Gesamtheit eindeutig und vollständig beschrieben sein.

Dies kann durch die Nutzung einer formalen Sprache, welche begriffliche Unschärfen und Mehrdeutigkeiten vermeidet und eine eindeutige Interpretation von Anweisungen zulässt, erreicht werden. Beispiele hierfür sind die mathematische Formelsprache oder Programmiersprachen.

2. Die einzelnen Schritte müssen zusammen praktisch durchführbar sein.

Dieses Merkmal verdeutlicht, dass jeder Algorithmus auf den Kontext seiner Realisierung bezogen ist. So kann beispielsweise ein Algorithmus zur Texterkennung nur eine*n für Nutzer*innen gewinnbringenden Output generieren, wenn er auch im Kontext von Texterkennungsprozessen eingesetzt wird. Im Kontext von Sortierungsverfahren z. B. verliert er an Legitimität.

3. Die Handlungsanweisung muss mechanisch ausführbar sein.

Dieses Merkmal ist nötig, damit ein Algorithmus unter unveränderten Voraussetzungen immer dasselbe Resultat erzielt. Das heißt, er kann maschinell interpretiert und ausgeführt werden (vgl. Stalder 2016: 167 f.).

Diese drei Charakteristika stellen nach Stalder (2016) die "kleinsten gemeinsamen Nenner" dar, durch die alle algorithmischen Systeme abstrakt beschrieben werden können.

\subsection{Big Data}

Die algorithmenbasierte Verarbeitung digitaler Daten verschiebt stetig die Grenzen des maschinell Machbaren (vgl. Patterson \& Gibson 2017: o. S.). Neben der Weiterentwicklung der algorithmischen Verarbeitungslogiken wird dieser Trend auch von den Daten, die verarbeitet werden, beeinflusst. Ein viel rezipiertes
Schlagwort, um die jüngste Entwicklung der globalen Datensphäre zu benennen, ist „Big Data“. Trotz anwachsender domänenübergreifender Prominenz des Begriffs findet sich keine weithin anerkannte Definition dafür. Ein auch noch heute oft zitiertes Modell zur Charakterisierung des „Big Data“-Begriffs ist das Modell der 3 Vs nach Laney (2001, zit. n. Kitchin 2014: 68): Big Data zeichnet sich demnach aus durch:

1. Volume: Die Menge der Daten ist groß.

2. Velocity: Die Geschwindigkeit, in der Daten generiert und geteilt werden, ist hoch.

3. Variety: Die Daten sind divers im Hinblick auf Datentyp, Strukturierung der Daten und deren zeitliche und räumliche Referenz.

In welchen Dimensionen diese Entwicklung von statten geht, lässt sich anhand folgender Zahlen verdeutlichen: 2018 wurde die Größe der globalen Datensphäre auf etwa 33 Zettabyte geschätzt ( 1 Zettabyte = eine Milliarde Terabytes). Diese Datenmenge soll bis zum Jahr 2025 bereits auf 175 Zettabyte anwachsen (vgl. Reinsel et al. 2018: 3). Die Zahlen verdeutlichen einerseits die enorme Menge (volume) der global bereits vorliegenden Daten und andererseits die hohe Geschwindigkeit (velocity), in der die Daten generiert werden. Damit geht auch ein Anstieg in der Diversität (variety) der erhobenen Daten einher (Kitchin 2014: 76). Mit der dauerhaften Generierung von Big Data werden neben gezielt erhobenen Daten, sogenannter "captured data", auch immer mehr "exhaust data" (Kitchin 2014: 6) erhoben, welche zunächst nicht im Zentrum der vorgenommenen Datenerhebung stehen, sondern als Nebenprodukt anfallen. Das heißt aber nicht, dass diese exhaust data nicht auch zur Gewinnung weiterführender Informationen über den eigentlichen Zweck der Dateninfrastruktur hinaus fruchtbar gemacht werden können. Ein elektronisches Kassensystem im Supermarkt ist beispielsweise zunächst dazu konzipiert, die eingekauften Waren aufzusummieren. Nebenbei werden jedoch auch Daten erfasst, die Aussagen über den Warenbestand im Markt, das Einkaufsverhalten von Kund*innen oder die Produktivität der Kassierer*innen zulassen (vgl. Kitchin 2014: 6).

\subsection{Data Science, Neuronale Netze und Maschi- nelles Lernen}

Das Forschungs- und Anwendungsfeld der Data Science widmet sich der Nutzung solcher Daten zur Gewinnung neuer Informationen und den dafür nötigen Methoden und Prozessen. Ziel ist die Generierung von verwertbaren Smart Data durch Verfeinerung und Analyse von Big Data-Rohdaten mithilfe automatisierter algorithmischer Auswertungsmethoden (vgl. Swarat 2018: 14). Als Schnittstellendiszip- 
lin zwischen Informatik, Mathematik und Statistik ist Data Science ein zentraler Treiber bei der Entwicklung komplexer Algorithmusassemblagen, welche zunehmend als neuronale Netze entworfen werden und zu maschinellem Lernen (ML) fähig sind. Beim maschinellen Lernen optimiert eine Algorithmusassemblage die eigene Funktionsweise in Abhängigkeit von den verarbeiteten Daten durch rekursive Operationen (Levermann 2018: 36). Die Algorithmen "lernen“ also durch wiederholtes Analysieren die Struktur der Datenassemblagen kennen und extrahieren daraus Strukturen (Patterson \& Gibson 2017: o. S.). So werden Korrelationen zwischen zwei Parametern beispielsweise mithilfe linearer Regressionen und komplexer Entscheidungssysteme unter Entwicklung digitaler neuronaler Netze modelliert. Bei komplexeren neuronalen Netzen spricht man von Deep Learning. Eine explizite Abgrenzung der beiden Begriffe findet sich allerdings nicht. Patterson \& Gibson fassen neuronale Netze mit mehr als zwei Schichten an Neuronen, also mehr als zwei Ebenen innerhalb des algorithmischen Entscheidungsbaumes, unter dem Begriff „Deep-Learning-Systeme“ zusammen.

Wenn wir im Folgenden von Algorithmen sprechen, bedienen wir uns dem in den Sozialwissenschaften vorherrschenden Verständnis von Algorithmen und meinen komplexe Algorithmusassemblagen im Sinne maschinell lernender neuronaler Netze.

\section{Algorithmen und soziokulturelle sowie räumliche Implikationen}

Stalder (2016) entwirft in seinem Buch „Kultur der Digitalität" eine Zeitdiagnose, in der Algorithmizität neben Referentialität und Gemeinschaftlichkeit eines von drei zentralen kulturellen Prinzipien darstellt. Die drei Prinzipen charakterisieren kulturelle Prozesse und sind aufgrund vielfältiger Verstrickungen nicht trennscharf voneinander abzugrenzen. „Referentialität“ beschreibt eine Methode zur Einschreibung Einzelner in kulturelle Prozesse. Dabei werden Bezüge zwischen eigenen und bereits bestehenden kulturellen Artefakten hergestellt und damit in ein kulturell bedeutsames Gefüge eingegliedert. Als „Gemeinschaftlichkeit“ wird der Austausch mit anderen zur Orientierung innerhalb komplexer Umwelten, wie sie sich in einer Kultur der Digitalität vor dem Hintergrund großer Informationsfluten konstituieren, beschrieben.

\subsection{Algorithmuskulturen der Digitalität}

In Bezug auf Stalder und weiteren Autoren fragt Levermann (2018), ob „angesichts der Bedeutung und ihres konstitutiven Charakters, die Algorithmen in einer Infosphäre haben, [...] nicht spezifischer von einer Algorithmuskultur der Digitalität gesprochen werden sollte" (ebd.: 40). In unserem Beitrag nutzen wir daher diesen Begriff, sprechen aber im Sinne von Seyfert und Roberge (2017) aufgrund ihrer vielfältigen kulturellen Entfaltungen bewusst von Algorithmuskulturen im Plural. Dabei geht es um die „Untersuchung bedeutsamer performativer Effekte, die mit algorithmischen Zugriffen auf die Welt einhergehen" (Seyfert \& Roberge 2017: 14). Im Zentrum stehen dabei Fragen wie: „Was tun Algorithmen, was bringen sie kulturell hervor? Wie generieren sie Sinn aus ihren Umgebungen und den verschiedenen Kategorien, die Menschen nutzen, um die Algorithmen zu deuten?" (ebd.). Hierzu bedarf es einer Ausweitung des utilitaristischen Verständnisses von Algorithmen, da diese nicht kausal auf Kultur einwirken, sondern deren Dispositive bestimmen (vgl. Hartmann 2018: 152). Auf diese reagieren Personen, je nach Profession und Erfahrung, sehr unterschiedlich. Dementsprechend variiert auch der Bedeutungsrahmen dessen, was mit Kultur gemeint ist: Eine Gruppe von Social-MediaInfluencer*innen erschafft eine andere und vermutlich spezifischere Art der Algorithmuskultur (z. B. in der Diskussion der Frage, wie Beiträge auf Plattformen eine möglichst hohe Reichweite bekommen) als Millionen von Pendler*innen, die sich jeden Morgen auf den Algorithmus von GoogleMaps verlassen, um den (scheinbar) schnellsten Weg zur Arbeit zu finden.

Darüber hinaus generieren Algorithmen auch kulturelle Identitäten (vgl. Rauer 2017: 193). Dies tun sie in unterschiedlichsten Formen. Als digitale Gatekeeper kuratieren sie kulturelle Artefakte und nehmen damit Rollen menschlicher Kurator*innen, wie Kunstmäzen*innen oder Zeitungskritiker*innen ein (Seyfert \& Roberge 2017: 18). Durch die Analyse vorhandener Nutzer*innendaten (Stichwort: Big Data) werden zudem Voraussagen getroffen, welche Informationen ein*e Benutzer*in auffinden möchte. Mit diesen Zuschreibungen werden meist intransparente Informationsangebote und Trending-Listen konstruiert. Empfehlungen solcher Trends innerhalb digitaler Dienste mithilfe von Vorschlags-Systemen können zur Ausbildung von Filterblasen und Echokammern beitragen (vgl. Gillespie 2017: 87 f.).

Algorithmusnetzwerke bewegen sich also in Form diverser Verkettungen unterschiedlicher mathematischer Mess- und Rechentechniken quer durch verschiedene soziale, ökonomische und politische Sphären. Dabei entwickeln sie unter Einfluss von ML-Mechanismen, wie in der Einleitung aufgezeigt, lebhafte Eigendynamiken, deren exakte Architektur und Funktionsweise meist nur lückenhaft oder intransparent kommuniziert werden. Häufige Folgen 
dieser Unschärfen sind Mystifizierungen oder die Reduktion von Algorithmen auf Black Boxes (vgl. Seyfert \& Roberge 2017: 21; Levermann 2018: 31).

\subsection{Algorithmen, Mensch \& Gesellschaft}

Die komplexe Verstrickung von Algorithmen, Mensch und Gesellschaft macht es schwer, klare Akteursgrenzen innerhalb der soziotechnischen Handlungsketten zu benennen (vgl. Rauer 2017: 191). Dies hat zur Folge, dass Algorithmuskulturen aus diversen soziologischen Perspektiven heraus entwickelt und beschrieben werden. Neben handlungs- und praxistheoretischen Ansätzen (u.a. Allert et al. 2017; Hörning 2017) finden sich Ansätze der Akteur-Netzwerk-Theorie (ANT), die ergänzend zu einer subjektorientierten Ausrichtung auch Objekte und Technologien als Akteure mit einbezieht (vgl. Latour 1999). Je nach theoretischer Schwerpunktsetzung werden Algorithmen damit verschiedene Rollen innerhalb soziotechnischer Systeme zugestanden.

Aus praxistheoretischer Sicht können Algorithmen als eine Unterform allgemeiner performativer Praktiken konzeptualisiert werden (vgl. Rauer 2017: 193), da sie sich ebenso wie Rituale, Narrative oder andere symbolische Handlungen durch rekursive, stark verinnerlichte Routinen auszeichnen (vgl. Seyfert \& Roberge 2017: 23). Handlungstheoretisch argumentiert fungieren Algorithmen als Transformatoren subjektiver Relationen (vgl. Allert \& Richter 2017: 25), indem sie und ihre Logiken durchgehend in menschliche Handlungen eingewoben sind (vgl. Hörning 2017: 70). Sie sind dabei „Nukleus einer technisch vermittelten Rahmung, Kontrolle und Kuratierung sozialen Handelns" (Dolata 2019: 198). Durch diese komplexe Verstrickung von Handlungsträgerschaft zwischen menschlichen und nicht-menschlichen Akteur*innen können die Grenzen der Handlungsträgerschaft selbst in Bewegung geraten (vgl. Rauer 2017: 210). Besonders deutlich wird das beispielsweise bei der juristischen Verhandlung von Fällen, in denen algorithmische Entscheidungssysteme involviert sind. Rauer (2017: 202 ff.) illustriert dies am Fall Ramstein, bei dem die Bundesrepublik Deutschland als Verantwortungsträgerin für Tötungen von Zivilist*innen durch amerikanische Drohnen im Jemen außerhalb von Kriegsgebieten angeklagt wurde. Obwohl keine deutschen Staatsbürger*innen an den Tötungen beteiligt waren und sich die Tötungen nicht auf deutschem Territorium ereigneten, akzeptierte das Kölner Verwaltungsgericht 2015 eine der Klagen jemenitischer Angehöriger von Drohnenopfern. Grund dafür ist die Tatsache, dass ein entscheidender Bestandteil der algorithmisch gesteuerten Infrastruktur des amerikanischen Drohnenprogrammes auf der amerikanischen Militärbasis in Ramstein stationiert ist. Das Beispiel zeigt, wie algorithmische Interaktionstechnologien die Relation zwischen menschlichem und nicht-menschlichem Handeln verschieben und die Beantwortung von Fragen nach der Verantwortlichkeit erschweren. Zudem verweist das Beispiel auf die Bedeutung des physisch-materiellen Raums für digitale Handlungspraktiken, die sich nicht einfach im virtuellen Raum „aufösen“, sondern sich ganz konkret in der Frage nach dem räumlichen Ursache-Wirkungs-Prinzip algorithmischer Entscheidungen widerspiegeln, was wir in Kap. 3.3 ausführlich besprechen.

Der ko-konstitutive Charakter algorithmischer Systeme und der dynamische Fluss von Handlungsträgerschaft erschweren die diskrete Kategorisierung von Rollen, die Algorithmen in soziokulturellen Prozessen einnehmen. Als Orientierungshilfe zur Charakterisierung verschiedener Rollen, die Algorithmen speziell aus geographischer Perspektive einnehmen können, skizzieren wir aufbauend auf den vorausgegangenen Ausführungen einen Vorschlag. An ihm können sich Lehrende für die Planung und Analyse von Unterrichtseinheiten orientieren. Dies bezieht sich insbesondere auf die Gestaltung von Lernumgebungen und die darin genutzten Aufgaben. Das Schema (vgl. Abb. 1) spannt ein Kontinuum zwischen Technikund Sozialdeterminismus auf. Die Betrachtung von Algorithmen als Werkzeuge in der Geographie bildet dabei eine stark sozialdeterministisch orientierte Sichtweise ab, während die Rolle von Algorithmen als Konfiguratoren räumlicher Praxis maßgeblich technikdeterministische Sichtweisen auf Algorithmen umfasst. Algorithmen als Teil handlungstragender Infrastruktur sind im Schema zwischen den beiden Rollen verortet und erkennen sowohl menschliche als auch algorithmische Handlungsträger*innen als teilautonome Subjekte in soziomateriellen Gefügen an. Die drei Bereiche sind nicht als strikt abgegrenzte Klassifikationen, sondern lediglich als Wegmarken zur

\section{Algorithmen als Werkzeuge in der Geographie}

\section{Algorithmen als Teil handlungstragender Infrastruktur}

Algorithmen als Konfiguratoren räumlicher Praxis

Sozialdeterminismus

Technikdeterminismus 
Orientierung innerhalb des Kontinuums zu begreifen. Je nach gewählter Perspektive nimmt der Einfluss von Algorithmen auf menschliches Handeln und soziale Praxis also von links nach rechts zu.

Wie Algorithmen innerhalb des geographischen Gefüges verortet werden, ist stets situativ und akteursbezogen zu bewerten. Während die Entwickler*innen algorithmischer Systeme diese vermutlich vorwiegend als Werkzeuge betrachten würden, können diese von Nutzer*innen als Interaktionspartner und Handlungsträger wahrgenommen werden. Nachfolgend werden wir uns den drei Rollenbeschreibungen unter Rückgriff auf konkrete geographische Beispiele annähern.

\subsection{Algorithmen und räumliche Implikationen}

In der Geographie hat sich der Begriff des „Digital Turns" etabliert, in dessen Zuge der Einfluss der Digitalität auf Raum und Räumlichkeit für die Geographie thematisiert wird. Gleichzeitig konstituierten die vielfältigen Partizipationsmöglichkeiten, die die Digitalität in Bezug auf die Geographie ermöglicht, eine sogenannte "Neogeographie“ (Boeckler 2014). Gleichwohl ist es in den letzten Jahren zu einer Verstetigung dieses neuen Wissenschaftsbereichs innerhalb der Geographie gekommen. Dies wird nicht nur durch die stetig steigende Anzahl von Artikeln deutlich, sondern auch durch die Gründung eines von der DFG geförderten Netzwerks „Digitale Geographien“ im Jahr 2018, die Etablierung von Zeitschriften, wie z. B. „Digital Geography and Society“, die 2020 erstmals erschienen ist, und die zunehmenden Umwidmungen oder Neuausschreibungen von Professuren mit der Denomination „Digitale Geographie“.

Wie sich nun Digitalität bzw. Software auf den Raum als zentralen Forschungsgegenstand der Geographie auswirkt, beschreiben Kitchin \& Dodge (2011): „Software [...] [works] across geographic scales and times to produce diverse spatialities. From this perspective, society, space, and time are co-constitutiveprocesses that are at once social, spatial, and temporal in nature and produce diverse spatialities" (ebd.: 13). Dieses Zitat verdeutlicht, dass neben neuen Verständnissen von Raum und Räumlichkeit die andauernde Digitalisierung der Gesellschaft auch eine Transformation sozialer Praktiken zur Raumerschließung, -wahrnehmung, -konstruktion und -produktion mit sich bringt. Damit wirkt sie sich grundlegend auf die Geographie als wissenschaftliche Disziplin sowie auf zahlreiche ihrer Teildisziplinen (kulturelle Geographie, Geoinformatik, Geomedienbildung, etc.) und deren epistemologische Ausrichtung und Methoden aus (vgl. Ash et al. 2019; Felgenhauer \& Gäbler 2019).

Die steigende Relevanz von Algorithmiziät als zentrales kulturelles Prinzip und die damit einherge- hende Diffusion von Handlungsträgerschaft zwischen menschlichen und nicht-menschlichen algorithmischen Aktanten werfen Fragen nach deren Rolle in räumlich-konstitutiven Handlungsgefügen auf. In diesem Kontext entwickelten sich in den vergangenen Jahren neue Sichtweisen auf Räumlichkeit, die Algorithmen auf verschiedene Arten innerhalb sozialräumlicher Gefüge verorten. Dies impliziert, dass es zur Erfassung und Vermessung von Raum im Kontext von Algorithmuskulturen einer multiperspektivisch orientierten Sicht auf Raum bedarf, anstatt der singulären Betrachtung rein positivistischer oder rein handlungszentrierter Raumperspektiven. Zur Verdeutlichung der verschiedenen Rollen von Algorithmen innerhalb sozialräumlicher Gefüge werden die drei Rollenbeschreibungen aus dem vorhergehenden Punkt aufgegriffen und in räumlichen Kontexten exemplarisch vorgestellt.

\section{Algorithmen als Werkzeuge in der Geographie}

Algorithmen und ML-Systeme halten unter anderem als Hilfsmittel zur Auswertung von Satellitendaten Einzug in die geographische Praxis (vgl. Ash et al. 2019: 4; Brandt et al. 2020). Mithilfe hoch aufgelöster Aufnahmen lassen sich Bilderkennungssysteme für die Bestimmung verschiedener Landschaftsformen und Oberflächenbedeckungen trainieren (vgl. Brandt et al. 2020: 79). Sie treten damit an die Stelle manueller Klassifikationen durch Geograph*innen und werden zu einem entscheidenden Bestandteil des geographischen Erkenntnisgewinns. Im Oktober 2020 sorgte die ML-gestützte Entdeckung von Bäumen in der westafrikanischen Sahara und Sahelzone für Schlagzeilen in wissenschaftlichen Medien. Aufgrund der verstreuten Lage von Bäumen in ariden Gebieten ist deren weltweite Anzahl, Lage und Größe für die manuelle Klassifizierung auf Satellitenbildern kaum erfassbar (ebd.: 78). ML-Systeme hingegen können für diese kleinteilige Erfassung trainiert werden. Das Forscher*innen-Team publizierte die Entdeckung der Bäume in der Zeitschrift Nature unter der Schlagzeile „An unexpectedly large count of trees in the West African Sahara and Sahel" (ebd.). Die mit Hilfe von Algorithmen gewonnene Erkenntnis über die unerwartet große Anzahl an Bäumen verändert jedoch nicht nur das Bild, das Forscher*innen über den physisch-materiellen Raum haben, sie verändert auch die Sicht auf das ökologische Wirkungsgefüge vor Ort. So stellen Bäume in ariden Biomen essentielle Faktoren für das Überleben der Biodiversität in Flora und Fauna dar. Sie haben positive Auswirkungen auf die Bodenbeschaffenheit, sind Bestandteil von Wasser- und Nährstoffzyklen, haben Einfluss auf das Klima und fungieren als Kohlenstoffspeicher (vgl. ebd.: 78). 
Bezüglich des Rollencharakters von Algorithmen als Werkzeuge in der Geographie müssen wir an dieser Stelle relativieren, dass sich mit der Nutzung solch automatisierter Bilderkennungssoftware natürlich auch die geographische Forschungspraxis ändert und Algorithmen somit auch als Handlungsträger verortet werden können. Im Kontext des aufgezeigten Fallbeispiels stellt der Algorithmus jedoch in erster Linie ein erfolgreiches und neues Werkzeug dar, das den Prozess der Klassifizierung von Satellitenbildern automatisiert und verbessert.

\section{Algorithmen als Teil handlungstragender Infra- struktur}

Wie das Beispiel Rauers (2017) zu den Drohneneinsätzen im Jemen zeigte, ermöglichen digitale Technologien die Ausbildung von Infrastrukturen über weite Distanzen. Sie ermöglichen eine Aufweitung von Handlungsvollzügen über große Distanzen hinweg und lassen sich somit als handlungstragend bezeichnen. Dennoch sind die Akteursnetzwerke dahingehend lokalisiert, dass die Zugangspunkte der Netzwerke geographisch verortbar sind. Damit erscheinen und wirken die dementsprechenden Infrastrukturen gleichzeitig global und lokal. Ein weiteres Merkmal der Infrastruktur des Drohnenprogramms ist ihre Abhängigkeit von Software: Kitchin und Dodge (2011) entwickeln zur Beschreibung solch dyadischer Systeme, die erst durch ihre Software und ihre materielle Repräsentation im physischen Raum gegenständlich werden, den Begriff „Code/Space“: „Code/space occurs when software and the spatiality of everyday life become mutually constituted, that is, produced through one another" (ebd: 16). Als alltägliches Beispiel führen sie die Check-In-Hallen von Flughäfen an. Analog zur Supermarktkasse (vgl. 2.3.) wird die Funktionalität der Check-In-Halle erst durch die materielle Infrastruktur und die entsprechende Software in Kombination miteinander produziert. Ist eine der beiden Hälften des dyadischen Systems ausgeschaltet oder defekt, so kann der Zusammenhang Code/ Space nicht hergestellt werden (vgl. ebd: 18). Dadurch grenzen Kitchin und Dodge (2011) Code/Spaces von sogenannten "Coded Spaces" ab, welche zwar durch Software beeinflusst werden, deren Räumlichkeiten aber in gleicher oder ähnlicher Weise auch ohne den Einfluss von Software produziert werden können. Als Beispiel führen sie dabei foliengestützte Vorträge an: Zwar kann die Präsentation digitaler Folien während eines Vortrags zum Verständnis der Inhalte im Publikum beitragen, der Vortrag und dessen Inhalte können aber auch ohne Folien vermittelt und die Räumlichkeit damit weiterhin produziert werden. „Space" beschreibt folglich nicht lediglich die mate- rielle Gegebenheit, in der eine Handlung ausgeführt wird, sondern begreift Räumlichkeit als ein "event or a doing - a set of unfolding practices that lack a secure ontology - rather than a container or a plane or a predetermined social production that is ontologically fixed" (ebd.: 16). Code/ Spaces skalieren dynamisch und befinden sich in einem stetigen Zustand der Neukonfiguration. Während der Durchführung einer Transaktion an einem Geldautomaten wird z. B. eine Verbindung zum zentralen Datenserver der Bank aufgebaut, der im Hauptsitz der Bank lokalisiert sein kann, während sich der Automat selbst in einer Seitenstraße befindet (vgl. ebd. 2011: 78). In diesem Moment wird die Handlungsträgerschaft für kurze Zeit räumlich aufgeweitet und kontrahiert anschließend wieder.

Dass Code explizit zur Produktion von Raum geschrieben wird und algorithmische Systeme als Handlungsträger in räumliche Planungsprozesse Einzug halten, wird besonders am Beispiel von Smart Cities deutlich. Vor dem Hintergrund des Smart-City-Konzepts werden Planstädte und Stadtplanungskonzepte entwickelt, in der Informations- und Kommunikationstechnologien eng mit städtischer Infrastruktur verwoben sind. Algorithmen halten damit zunehmend Einzug in die Verwaltung von Raumnutzungsdaten innerhalb der Stadt (vgl. Resch \& Szell 2019). Analog zu Code/Space konstituieren sich damit Raum und Algorithmen gegenseitig. Algorithmen und deren Logiken werden zu ubiquitären Handlungsträgern und Teil städtepolitischer Entscheidungsfindung in Smart Cities, wenn z. B. Verkehrsinfrastruktur anhand von automatisch erfassten Daten des PKW-Verkehrs „optimiert" wird (Greenfield 2014). Als Teil handlungstragender Infrastruktur sind sie in diesem Sinne Teil dyadischer Systeme aus physisch-materiellen Entitäten und Software, die in ihrer Kombination Räumlichkeit produzieren. Diesbezüglich wird ihnen eine teilautonome Handlungsträgerschaft zuteil, welche sich im raumkonstituierenden Handlungsvollzug entfaltet. Dabei zwingen Algorithmen Code/Spaces nicht zwangsläufig ihre Logiken auf. Der Bezahlvorgang an der Ladenkasse selbst änderte sich durch die Digitalisierung von Bezahlsystemen kaum: Die Kund*innen bezahlen in bar oder mit EC-Karte einen Betrag, der zuvor von der Kasse (oder den Kassierenden) errechnet wurde. Im Fall von Smart Cities hingegen werden algorithmische Logiken zunehmend zu Gestaltungsprinzipien des städtischen Raums und damit zu Moderatoren städtischen Lebens. Dies fasst Roche (2017: 661) mit Bezug auf Konzepte von „BigGeo-Data", die ebenfalls algorithmisch ausgewertet werden, zusammen: "Digital technology, especially geo-technology, is becoming consubstantial to urban materiality, and in this context, map interfaces play a 
privileged role in indexing geographic knowledge and expertise." Dies verdeutlicht den fließenden Übergang von Algorithmen als Teil handlungstragender Infrastruktur zu Konfiguratoren raumbezogener Praxis.

\section{Algorithmen als Konfiguratoren raumbezogener Praktiken}

Die Dominanz von Algorithmizität im städtischen Umfeld als Konfiguratoren raumbezogener Praktiken wird auch anhand von Protestkulturen deutlich. So werden beispielsweise Bots, also Computerprogramme, die eigenständig im Internet agieren und kommunizieren (Oberer 2019: 311), darauf programmiert, digitale Hinweise von Bürger*innen auf Fahrscheinkontrollen zu sammeln und über ein eigenes TwitterKonto weiterzuverbreiten. Mithilfe der Hinweise, die durch die Bots eine enorme Reichweite erlangen, können andere Fahrgäste öffentlicher Verkehrsmittel Fahrscheinkontrollen annähernd lokalisieren und gegebenenfalls umgehen (vgl. Krempl 2020: o. S.). Der Bot illustriert exemplarisch, wie Algorithmen Einfluss auf Kommunikations- und Mobilitätspraktiken nehmen und diese transformieren können (vgl. Ash et al. 2019: 4).

Ein weiteres Beispiel zur Transformation raumbezogener Praktiken durch Algorithmizität stellen digitale Raumkonstruktionen durch Hashtags dar. Digitale Raumkonstruktionen legen den Fokus nicht auf das Handeln im Raum, wie es das Beispiel des oben genannten Twitter-Bots illustriert, sondern auf die digitale Re-, De- oder Konstruktion von Räumen, sowie deren Kommunikation und Wahrnehmung in sozialen Netzwerken. Indem Nutzer*innen beispielsweise Fotos eines Ortes in sozialen Netzwerken teilen und diesen mithilfe von Hashtags bestimmte Attribute zuordnen, konstruieren sie ein öffentlich zugängliches Bild des abgebildeten Raumes (vgl. Reithmeier et al. 2016: 282; Kanwischer \& Schlottmann 2017: 61). Ein Beispiel hierfür ist z. B. die Nutzung von ortsbezogenen Hashtags in Posts in den sozialen Netzwerken, wie z. B. \#frankfurt oder \#sylt. Die Benutzer*innen generieren, synthetisieren und interpretieren damit lokale Informationen. Dadurch werden neue Attribute der Bedeutung für einen bestimmten Raum erzeugt, die es vor einiger Zeit noch nicht gab. Hierbei beziehen sich die Nutzer*innen auf den gleichen ortsbezogenen Hashtag und Raum. Die Algorithmen der sozialen Netzwerke bestimmen jedoch letztendlich, welche - oftmals personalisierten - raumbezogenen Informationen wir wann und an welcher Stelle überhaupt bekommen. Somit entscheiden die Algorithmen, was die Grundlage unseres räumlichen Handelns wird (vgl. Reithmeier \& Dorsch 2021). Algorithmen sind in diesem Fall die entscheidenden Konfiguratoren raumbezogener Praxis. Weitere Beispiele sind z. B. die Verwendung von Karten-Mashups, der Einfluss von Empfehlungsportalen (digitale Mundpropaganda), der Google PageRank-Algorithmus im Hinblick auf räumliche Informationen und die Rolle von EchtzeitNavigationssystemen im Straßenverkehr.

Anhand der Beispiele wird deutlich, dass Algorithmen räumliche Routinen und Praktiken beeinflussen und sich somit als Konfiguratoren sozialer Praxis etabliert haben. Diese Rollenbeschreibung muss jedoch dahingehend relativiert werden, als es auch seitens der Algorithmen stetiger Transformation bedarf, um durch die veränderte Praxis nicht einem Verlust an Legitimität zu erliegen. So hat beispielsweise die softwaregestützte Überprüfung wissenschaftlicher Arbeiten nicht zwangsläufig eine Abnahme von Plagiaten, sondern eine Transformation des Plagiierens zur Folge (vgl. Seyfert \& Roberge 2017: 14). Um weiterhin Legitimität zu erfahren, muss der Algorithmus auf die veränderte Praktik angepasst werden. So wie Praktiken durch stabilisierende Wiederholungen und Habitualisierungen charakterisiert sind, so charakteristisch ist auch deren Transformation durch Irritation und Variation (vgl. Allert \& Asmussen 2017: 48). Beides ist Teil rekursiver Aushandlungen zwischen Algorithmen, Mensch und Gesellschaft. Geographische Bildung muss diese Aushandlungsprozesse in den Blick nehmen und Lernende dazu ermächtigen an ihnen zu partizipieren. Die Frage, wie dies gelingen kann, werden wir abschließend diskutieren.

\section{Die digitale Welt verstehen und mitge- stalten}

Wir konnten aufzeigen, dass die Algorithmuskulturen der Digitalität vielfältige gesellschaftliche und räumliche Implikationen haben. Im Bildungsbereich können diese Implikationen aus unterschiedlichen Perspektiven beobachtet und analysiert werden: Algorithmen als Werkzeuge, als Teil handlungstragender räumlicher Infrastrukturen oder als Konfiguratoren raumbezogener Praktiken.

Ein diesbezüglicher Blick in ausgewählte curriculare Dokumente und deren Kommentare ist jedoch ernüchternd. Hinsichtlich des neuen GW-Lehrplans für die Sekundarstufe I (vgl. Fridrich et al. 2019) wurden digitale Aspekte sehr allgemein thematisiert, wie z.B. hinsichtlich einer Orientierung in einer durch Digitalisierung geprägten Gesellschaft sowie in Bezug auf die Nutzung digitaler (Geo-)Medien in kritischer und kreativer Weise. Ähnlich sieht es bei den Bildungsstandards im Fach Geographie für den Mittleren Schulabschluss aus. Hier wurden im Rahmen der 10. Auflage in einigen Kapiteln „Erweiterungen und Aktualisie- 
rungen bezüglich des Beitrags unseres Faches zur Digitalisierung (...) vorgenommen" (Deutsche Gesellschaft für Geographie 2020: 3). Algorithmizität spielt diesbezüglich aber keine Rolle und die Aktualisierungen bezüglich Digitalisierung zeichnen sich insbesondere dadurch aus, dass analoge Medien nun um den Begriff „digitale“ erweitert wurden. Gleichwohl wird Algorithmizität im Positionspapier des Hochschulverbands für Geographiedidaktik (HGD) e.V. zur Bildung in einer durch Digitalisierung und Mediatisierung geprägten Welt thematisiert (HGD 2020). Diese Befunde verdeutlichen, dass das geographische Bildungssystem wieder einmal den fachlichen und technischen Entwicklungen hinterherhinkt.

Dies ist umso besorgniserregender, da die digitalen Fähigkeiten der ,Digital Natives', die in der digitalen Welt aufgewachsen sind und über die sozialen Netzwerke tagtäglich mit Algorithmuskulturen konfrontiert werden, nicht bei allen Schüler*innen und Studierenden stark ausgeprägt sind. Dies verdeutlichen die Ergebnisse der International Computer and Information Literacy Study 2018. Ein Drittel der Schüler*innen in Deutschland kann kaum oder nur schlecht mit einem Computer oder vergleichbarer Technik umgehen (vgl. Eickelmann et al. 2018: 281). Birgit Eickelmann, die für den deutschen Teil der Studie verantwortlich war, bringt es in einem Interview mit dem Spiegel auf den Punkt: „Diese Schüler können eigentlich nur Links anklicken und ihr Handy streicheln" (Unterberg 2019). Hiermit wird auch ein klarer Bildungsauftrag an das Fach Geographie herangetragen: In der geographischen Bildung an Schule und Hochschule müssen Lerngelegenheiten geschaffen werden, die es den Schüler*innen und Studierenden ermöglichen, Fähigkeiten zu entwickeln, mündig zu agieren und somit Entwicklungen zu hinterfragen und die digitale Gesellschaft verantwortungsvoll und kreativ zu gestalten.

Wir stellen im Folgenden vielversprechende Ansätze vor, die eine mündige Auseinandersetzung mit Digitalität bzw. Algorithmizität fördern. Abb. 1 dient uns dabei als Analyseschema, um die Konzepte hinsichtlich des in ihnen fokussierten Algorithmenverständnisses zwischen Sozialdeterminismus und Technikdeterminismus strukturieren zu können. Uns ist bewusst, dass die Ansätze in der Regel für eine umfassende Medienbildung stehen, dennoch lassen sich insbesondere in den verwendeten Beispielen Tendenzen im Hinblick auf das Rollenverständnis ableiten.

Fuchs et al. (2021) ergänzen das Spatial Citizenship-Konzept (Jekel et al. 2015; Schulze et al. 2015) um Aspekte, die in Algorithmuskulturen relevant sind. Im Zentrum steht dabei die These, dass digitale Geomedien halbautonome, algorithmische Systeme sind, die Nutzer*innen in ihren Partizipationsbe- strebungen beeinflussen können. Hieraus leiten die Autoren Kompetenzen ab, die u.a. darauf abzielen, dass Lernende reflektiert mit algorithmischen Kommunikationssystemen (z. B. Social Bots) interagieren können. Der erweiterte Spatial-Citizenship-Ansatz fokussiert sowohl die Reflexion sozialer Praktiken also auch die Förderung technischer Kompetenzen im Umgang mit Algorithmen. So sollen Lernende z. B. in Interaktion mit algorithmischen Systemen analytische Operationen zur Lösung räumlicher Probleme und zur Auswertung GIS-basierter Datenanalysen durchführen können. Das Konzept deckt somit die gesamte Breite des Analyseschemas ab.

Ein Ansatz, der vorrangig die Rolle von Algorithmen als Konfiguratoren sozialer Praxis in den Blick nimmt, stammt von Richter \& Allert (2017). Indem Algorithmen als Handlungs- und Entscheidungsträger, z. B. in sozialen Netzwerken, die Lernenden mit Unbestimmtheit konfrontieren, bieten sie Anlässe zur kreativen Auseinandersetzung und stoßen damit Lernprozesse an (Allert \& Richter 2017: 28). Dies kann sich im besten Fall darin äußern, dass Lernende „poetische Spielzüge“ durchführen, welche die formalisierten Regeln des praktischen Vollzugs algorithmischer Systeme zumindest hinterfragen (Richter \& Allert 2017: 254). Doch stellen die Autor*innen ernüchternd fest, dass Widerspruch und die Zweckentfremdung gegenüber algorithmischen Systemen insofern unmöglich ist, ,indem sie jedwede Handlung entsprechend der in ihnen realisierten Interpretation der praktischen Situation auslegen" (Richter \& Allert 2017: 257).

Dorsch und Kanwischer (2020) stellen die Kreativität in den größeren Kanon einer mündigkeitsorientierten Bildung, die aus den drei Dimensionen „Reflexivität“, „Sich-seiner-selbst-bewusst-Sein“ sowie „Autonomie" besteht. Alle drei Dimensionen werden durch die Digitalität und insbesondere durch Algorithmen zum einen beeinflusst, zum anderen dienen die Fähigkeiten auch dazu, um in einer Kultur der Digitalität mündig agieren zu können. Den Autoren kommt es zwar darauf an, dass Lernende digitale Medien einerseits selbstbestimmt für ihre Zwecke nutzen können sollen (z.B. indem sie online eine Initiative gründen). Andererseits lehnen sie eine einseitig technisch-instrumentelle Ausbildung der Lernenden ab, die Algorithmen rein als technische Werkzeuge betrachtet (Dorsch \& Kanwischer 2020: 37-38).

Schiefner-Rohs betont in einem Aufsatz über blinde Flecken der Medienpädagogik, in dem Algorithmizität ansonsten eine untergeordnete Rolle spielt, die Bedeutung von Algorithmen als Konfiguratoren schulischer Organisationspraktiken. In Form von digitalen Tools zur Innovation von Unterrichtssituationen (z. B. Learning-Analytics-Software) können Algorithmen 
Lehrkräften als Werkzeuge dienen (Schiefner-Rohs 2017: 160). Die zugesprochene Rolle der Algorithmen im Aufsatz lässt sich also eher auf der sozialdeterministischen Seite des Schemas einordnen.

Ein Ansatz, der die Ubiquität des Digitalen in der „mediatisierten Gesellschaft“ beschreibt, ist die Strukturale Medienbildung (vgl. Jörissen \& Marotzki 2009). Der „Welt- und Selbstbezugs” des Individuums wird demzufolge durch das Digitale - und damit auch durch Algorithmizität - verändert, was sich wiederum auf die Gestaltung von Bildungsprozessen auswirkt (vgl. ebd.: 109). Jörissen und Marotzki verweisen dabei auf vier Dimensionen der Lebensweltorientierung, die auf Kant zurückgehen und die kritische Analyse und autonome Reflexion in den Fokus setzen. Im Fokus stehen vier Reflexionsgegenstände: (1) die Bedingungen und Grenzen des Wissens, z. B. Fragen nach Quellen von Wissen in einer mediatisierten Welt, (2) der Handlungsbezug als Frage nach Grundsätzen des eigenen Handelns, die sich in sozialen Kontexten ergeben, z. B. Fragen nach Handlungsoptionen in einer mediatisierten Welt, (3) Grenzen als Grundstruktur von Bildung, z. B. Fragen danach, wie Kommunikationstechnologien Subjekte in konstitutiver Weise beeinflussen oder wie flexibel Grenzen sind sowie (4) die eigene Identität und die biographischen Bedingungen, z. B. die Überführung von Informationen in konsistente Wissenszusammenhänge und die eigene Verortung innerhalb dieser Zusammenhänge.

Wie diese Dimensionen als Analyseraster für die Untersuchung der oben beschriebenen Raumkonstruktionen durch Hashtags in sozialen Medien dienen können, zeigen Kanwischer und Schlottmann (2017). In Bezug auf den Handlungsbezug in Dimension (2) sollen Lernende z.B. reflektieren, inwiefern sie sich durch bestimmte Posts und den in ihnen verwendeten Hashtags zu Handlungen verleiten lassen, indem sie beispielsweise einen Raum aufsuchen oder meiden. Reithmeier und Dorsch (2021) ergänzen die Hashtaganalyse um den Aspekt der Algorithmizität, die sich z. B. darin äußert, dass Beiträge von den Erstellenden angepasst werden, um in den Newsfeeds der sozialen Medien besser platziert zu werden. Insbesondere die letzten beiden Arbeiten postulieren, genauso wie Gryl et al. (2020), das Ausprobieren von algorithmischen Systemen in Bildungskontexten. Nur auf diese Weise könnten Schüler*innen und Studierende die manipulative Wirkung der Algorithmen gezielt erfahren und reflektieren sowie mögliche Gegenstrategien ausprobieren. Die vorgestellten Arbeiten, die sich auf Jörissen und Marotzki (2009) beziehen, beschreiben Algorithmen vorrangig als Konfiguratoren raumbezogener Praktiken. Einzig in der Frage, wie ein Beitrag in den sozialen Medien gestaltet werden muss, um von Algorithmen favorisiert zu werden (Reithmeier \& Dorsch
2021), werden Algorithmen teils in ihrer Rolle als Werkzeug identifiziert und reflektiert.

Darüber hinaus wurden in den letzten Jahren Ansätze entwickelt, die nicht explizit, aber implizit Algorithmizität thematisieren und für den Themenbereich fruchtbar gemacht werden können. Hintermann et al. (2020) haben z. B. im Rahmen des Projektes MiDENTITY mediale Identitätskonstruktionen untersucht und ein "Toolkit for critical geographic media literacy" entwickelt, das Schüler*innen ermöglicht, "to develop a self-determined way of media use thereby reflecting on their own online practices of social in- and exclusion" (ebd.: 115). Um dieses Ziel zu erreichen, wurden fünf kategoriale Analyseschritte entwickelt, die die Lernenden auf Medieninhalte anwenden können: „Representation“ (z. B. Was wird gezeigt? Was fehlt?); „Regulation“ (z. B. Was wird als „wahr" oder „richtig" dargestellt?), „Signs“ (Wie werden diese "Wahrheiten“" produziert?), „Production“ (Welches Ziel hat der Medieninhalt? Wer verdient daran?); sowie „Identities/Target groups“ (Wer sind die Adressaten des Inhalts, welche Rolle spielen Echokammern bei der Konstruktion von Identität in Bezug auf den Medieninhalt?). Algorithmen kommen im Ansatz vorrangig als konstitutiver (und damit nicht beeinflussbarer) Bestandteil von Echokammern vor, die wiederum raumbezogene Praktiken hervorrufen können.

Felgenhauer und Gäbler (2019) betonen, dass die Thematisierung der sozialen und kulturellen Konstruktion digitaler Räumlichkeit in übergreifende Kompetenzmodelle, wie z. B. das österreichische Rahmenkonzept "digi.kompP" (vgl. Brandhofer et al. 2016) eingebettet werden muss. Gleichzeitig heben sie hervor, dass auch bei dem Themenfeld der digitalen Alltagskultur ,an vielen Stellen an eingeübte geographische Denkmuster und Theoriefiguren (wie etwa die Frage nach der räumlichen Verteilung von Infrastrukturen, Praktiken des Kartierens oder die mediale Repräsentation von Orten)" (Felgenhauer \& Gäbler 2019: 18) angeschlossen werden kann. Algorithmen werden in der Arbeit also sowohl in ihrer Rolle bezüglich raumbezogener Praktiken als auch als Teil handlungstragender Infrastruktur erfasst, deren Bedeutsamkeit für die Digitalität von den Schüler*innen erkannt werden soll.

Trotz der aufgezeigten Unterschiede in der Frage, in welcher Rolle Algorithmen jeweils fokussiert werden, ist den aufgezeigten Ansätzen gemein, dass Lernende innerhalb bestehender Algorithmuskulturen bestimmte Fähigkeiten benötigen, um an diesen partizipieren zu können. Diese reichen von Reflexivität, Selbstbestimmung bis hin zu Autonomie und Kreativität und lassen sich dem Begriffsfeld der Mündigkeit zuordnen. Dies erscheint zunächst als Widerspruch, 
da Algorithmen als festgelegte Handlungsanweisungen zunächst für kreative und mündige Praktiken nicht zugänglich erscheinen. Gillespie löst diesen Widerspruch auf: "Algorithms are not just what their designers make of them, or what they make of the information they process. They are also what we make of them day in and day out" (Gillespie 2014: 187). Wie wir also mit den Ergebnissen der algorithmischen Selektion umgehen, ob wir ihnen folgen oder ob wir versuchen unserer eigenen Sichtweise auf die Welt mehr Geltung zu verschaffen, indem wir vielleicht sogar versuchen Algorithmen zu beeinflussen, liegt an uns.

Vor dem Hintergrund, dass die Ausgestaltung der algorithmischen Systeme allein den Tech-Unternehmen überlassen bleibt, reichen die genannten Fähigkeiten aber mitunter nicht aus: Die Unternehmen bestimmen, wie Algorithmen zwischen wichtigen und unwichtigen Beiträgen unterscheiden, welche Produkte uns empfohlen werden und welche Nachrichten uns angezeigt werden. Es ist daher nicht verwunderlich, dass die Rufe nach einer „Digitalen Souveränität" lauter werden, die sich dadurch ausdrückt, dass Nutzer*innen möglichst die Kontrolle über die digitale Technologie erlangen oder zumindest deren Mechanismen verstehen. Neben der freien Verfügbarkeit und Veränderbarkeit von Software (Open Source) umfasst dies auch die Offenlegung der Codes von Algorithmen, um deren Entscheidungen nachvollziehen und ggf. dagegen Widerstand leisten zu können. Auf individueller Ebene bedeutet „Digitale Souveränität" aber auch die persönliche Kontrolle über Daten, Technologie und Software zu haben und hierfür entsprechend ausgebildet zu sein (vgl. Couture \& Toupin 2019: 2315 f.). Dabei geht es nicht darum, algorithmische Systeme zu verteufeln, da sie uns helfen die enormen Datenmengen zu sortieren und nicht darin zu „erblinden“ (Stalder 2017: o. S.). Insbesondere vor dem Hintergrund des anthropogen verursachten Klimawandels bedarf es intelligenter Technologien, die Energieversorgung, Mobilität und sonstige Infrastrukturen effizienter und ressourcenschonender machen. Nicht zuletzt in der Bekämpfung bzw. Nachverfolgung der Corona-Pandemie spielten Algorithmen eine wichtige Rolle (vgl. Chiusi et al. 2020). Doch entscheidend ist nach Stalder (2017: o.S.), dass die damit zusammenhängenden Konsultationen der algorithmischen Systeme transparent gemacht und in andere kollektive Entscheidungsprozesse eingebettet werden. Nur so kann gewährleistet werden, dass Algorithmen im wahrsten Sinn des Wortes berechenbar werden und wir verstehen können, warum uns zu bestimmten Zeiten und an bestimmten Orten z. B. Spotify bestimmte Musikvorschläge unterbreitet oder das Navi bestimmte Streckenvorschläge favorisiert.

\section{Förderhinweis}

Das diesem Artikel zugrundeliegende Vorhaben „Digi_Gap“ wird im Rahmen der gemeinsamen "Qualitätsoffensive Lehrerbildung" von Bund und Ländern mit Mitteln des Bundesministeriums für Bildung und Forschung unter dem Förderkennzeichen FKZ 01JA2025 gefördert. Die Verantwortung für den Inhalt dieser Veröffentlichung liegt bei den Autoren.

\section{Literatur}

Allert, H. \& M. Asmussen (2017): Bildung als produktive Verwicklung. In: Allert, H., M. Asmussen \& C. Richter (Hrsg.): Digitalität und Selbst - interdisziplinäre Perspektiven auf Subjektivierungs- und Bildungsprozesse. Transcript-Verlag, Bielefeld. S. 27-68.

Allert, H., M. Asmussen \& C. Richter (2017): Digitalität und Selbst: Einleitung. In: Allert, H., M. Asmussen \& C. Richter (Hrsg.): Digitalität und Selbst - interdisziplinäre Perspektiven auf Subjektivierungs- und Bildungsprozesse. Transcript-Verlag, Bielefeld. S. 9-26.

Allert, H. \& C. Richter (2017): Kultur der Digitalität statt digitaler Bildungsrevolution. In: Pädagogische Rundschau 71(1). S. 19-32.

Ash, J., R. Kitchin \& A. Leszcynski (2019): Introducing digital geographies. In: Ash, J., R. Kitchin \& A. Leszcynski (Hrsg.): Digital Geographies. SAGE, Los Angeles, London, New Delhi, Singapore, Washington DC, Melbourne. S. 1-10.

Boeckler, M. (2014): Neogeographie, Ortsmedien und der Ort der Geographie im digitalen Zeitalter. In: Geographische Rundschau 66(6). S. 4-10.

Brandhofer, G., A. Kohl, M. Miglbauer, T. Nárosy, J. Buchner, P. Groißböck, I. Lechner, J. Prinz, J. Prohaska, N. Zaynard, W. Fikisz, G. Futschek, K. Fuchs, P. Micheuz, H. Caba, W. Grossmann, A. Nußbaumer, G. Schwarz, F. Tranninger, R. Zoufal, H. Hammerl, H. Egger, H. Swaton, M. Steiner, A. Bachinger, S. Gabriel, C. Nosko, M. Schedler, P. Traxler, W. Wegscheider \& D. Wohlhart (2016): Das digi.kompP Kompetenzmodell. https:// www.virtuelle-ph.at/wp-content/uploads/2016/09/digi. kompP-Grafik-und-Deskriptoren-1.pdf (12.03.2021)

Brandt, M., C. J. Tucker, A. Kariryaa, K. Rasmussen, C. Abel, J. Small, J. Chave, L. V. Rasmussen, Hiernaux Pierre, A. A. Diouf, L. Kergoat, O. Mertz, C. Igel, F. Gieseke, J. Schöning, S. Li, K. Melocik, J. Meyer, S. Sinno, E. Romero, E. Glennie, A. Montagu, M. Dendoncker \& R. Fensholt (2020): An unexpectedly large count of trees in the West African Sahara and Sahel. In: Nature 587. S. 78-82.

Cardon, D. (2017): Den Algorithmus dekonstruieren: Vier Typen digitaler Informationsberechnung. In: Seyfert, R. \& J. Roberge (Hrsg.): Algorithmuskulturen. Über die rechnerische Konstruktion der Wirklichkeit. TranscriptVerlag, Bielefeld. S. 131-150. 
Chiusi, F., S. Fischer \& M. Spielkamp (2020): Automated Decision-Making Systems in the COVID-19 Pandemic: A European Perspective. Automating Society Report 2020, Special Issue. https://algorithmwatch.org/en/automating-society-2020-covid19/ (12.03.2021)

Couture, S. \& S. Toupin (2019): What does the notion of "sovereignty" mean when referring to the digital? In: New Media \& Society 21(10). S. 2305-2322.

Deutsche Gesellschaft für Geographie (2020): Bildungsstandards im Fach Geographie für den Mittleren Schulabschluss. 10. Aufl. Selbstverlag Deutsche Gesellschaft für Geographie, Bonn.

Dolata, U. (2019): Plattform-Regulierung. Koordination von Märkten und Kuratierung von Sozialität im Internet. In: Berliner Journal für Soziologie 29. S. 179-206.

Dorsch, C. \& D. Kanwischer (2020): Mündigkeit in einer Kultur der Digitalität: Geographische Bildung und "Spatial Citizenship”. In: Zeitschrift für Didaktik der Gesellschaftswissenschaften 11(1). S. 23-40.

Eickelmann, B., W. Bos, J. Gerick, F. Goldhammer, H. Schaumburg, K. Schwippert, M. Senkbeil \& J. Vahrenhold (Hrsg.) (2019): ICILS 2018 \#Deutschland. Computer- und informationsbezogene Kompetenzen von Schülerinnen und Schülern im zweiten internationalen Vergleich und Kompetenzen im Bereich Computational Thinking. Waxmann, Münster, New York.

Felgenhauer, T \& K. Gäbler (2019): Geographien digitaler Alltagskultur. Überlegungen zur Digitalisierung in Schule und Unterricht. In: GW-Unterricht 154(2). S. 5-20.

Fridrich, C., S. Hinsch, A. Koller, \& H. Pichler (2019): Der neue GW-Lehrplan für die Sekundarstufe I - ministerieller Auftrag, Herausforderungen, Struktur und erste Überlegungen. In: GW-Unterricht 154(2). S. 68-74.

Fuchs, L., D. Kanwischer \& C. Dorsch (2021): Algorithmic Cultures: A Missing Link of Spatial Citizenship Education. In: GI_Forum - Journal for Geographic Information Science. (In Druck)

Gillespie, T. (2017): \#trendingistrending: Wenn Algorithmen zu Kultur werden. In: Seyfert, R. \& J. Roberge (Hrsg.): Algorithmuskulturen. Über die rechnerische Konstruktion der Wirklichkeit. Transcript-Verlag, Bielefeld. S. 75-106.

Greenfield, A. (2014): The Smart City is predicited on an inappropriate model of optimization. In: derive 56 . S. 23-26.

Gryl, I., C. Dorsch, M. Lehner, J. Pokraka \& J. Zimmer (2020): Technologie, Medien, Mündigkeit: Integration autonomieförderlicher Haltungen und Kompetenzen in die fachliche Vermittlung. In: Beißwenger, M., B. Bulizek, I. Gryl \& F. Schacht (Hrsg,): Digitale Innovationen und Kompetenzen in der Lehramtsausbildung. Universitätsverlag Rhein-Ruhr, Duisburg. S. 121-146.

Hartmann, F. (2018): Medienmoderne Philosophie und Ästhetik. Spinger, Wiesbaden.

Hintermann, C., F. Bergmeister \& V. Kessel (2020): Critical Geographic Media Literacy in Geography Education:
Findings from the MiDENTITY Project in Austria. In: Journal of Geography 119(4). S. 115-126.

HGD (2000): Der Beitrag des Fachs Geographie zur Bildung in einer durch Digitalisierung und Mediatisierung geprägten Welt. Positionspapier des Hochschulverbands für Geographiedidaktik (HGD) e.V. http://geographiedidaktik.org/de/service/digitalisierung/ (12.03.2021)

Hörning, K. H. (2017): Wissen in digitalen Zeiten. In: Allert, H., M. Asmussen \& C. Richter (Hrsg.): Digitalität und Selbst - interdisziplinäre Perspektiven auf Subjektivierungs- und Bildungsprozesse. Transcript-Verlag, Bielefeld. S. 69-86.

Jekel, T., I. Gryl \& A. Oberrauch (2015): Education for Spatial Citizenship: Versuch einer Einordnung. In: GWUnterricht 137(1). S. 5-13.

Jörissen, B. \& W. Marotzki (2009): Medienbildung - Eine Einführung. Theorie - Methoden - Analysen. Klinkhardt, Bad Heilbrunn.

Kanwischer, D. \& A. Schlottmann (2017): Virale Raumkonstruktionen: Soziale Medien und Mündigkeit im Kontext gesellschaftswissenschaftlicher Medienbildung. In: Zeitschrift für Didaktik der Gesellschaftswissenschaften 8(2). S. 60-78.

Kitchin, R. (2014): The data revolution big data, open data, data infrastructures and their consequences. SAGE, Los Angeles.

Kitchin, R. \& M. Dodge (2011): Code/Space Software and Everyday Life. MIT Press, Cambridge.

Kowalski, R. (1979): Algorithm=Logic+Control. In: Communications of the ACM. 22(7). S. 424-436.

Krempl, S. (2020): Data Cities: Wie Hacktivisten Smart-City-Konzepte unterwandern. https://www.heise.de/news/ Data-Cities-Wie-Hacktivisten-Smart-City-Konzepte-unterwandern-4913295.html?utm_source=pocketnewtabglobal-de-DE (12.03.2021)

Laney, D. B. (2001): 3D Data Management: Controlling Data Volume, Velocity and Variety. META Group Research Note 6 .

Latour, B. (1999): Die Hoffnung der Pandora. Untersuchungen zur Wirklichkeit der Wissenschaft. Suhrkamp, Frankfurt.

Levermann, T. (2018): Wie Algorithmen eine Kultur der Digitalität konstituieren: Über die kulturelle Wirkmacht automatisierter Handlungsanweisungen in der Infosphäre. In: Journal für korporative Kommunikation 2. S. 31-42.

Oberer B., ErkollarA. \&A. Stein (2019): Social Bots-Act Like a Human, Think Likea Bot. In: StumpfM. (Hrsg.): Digitalisierung und Kommunikation. Europäische Kulturen in der Wirtschafts-kommunikation 31. Springer VS, Wiesbaden.

Patterson, J. \& A. Gibson (2017): Deep Learning. O’Reilly Media, Sebastopol.

Rauer, V. (2017): Drohnen: zur Materialisierung von Algorithmen. In: Seyfert, R. \& J. Roberge (Hrsg.): Algorithmuskulturen. Über die rechnerische Konstruktion der Wirklichkeit. Transcript-Verlag, Bielefeld. S. 189-214. 
Reinsel, D., J. Gantz \& J. Rydning (2018): The Digitization of theworld:From Edge to Core.AnIDCWhitePaper.https:// www.seagate.com/files/www-content/our-story/trends/ files/idcseagate-dataage-whitepaper.pdf

Reithmeier, C., K. Buschbaum, A. Blitz \& D. Kanwischer (2016): "Heaven. \#shopping \#Frankfurt \#weekend \#joy" - Hashtags, constructions of space, and geography education. - GI_Forum 4(1). S. 282-294.

Reithmeier, C. \& C. Dorsch (2021): Soziale Medien. In: Bork-Hüffer, T., H. Füller \& T. Straube (Hrsg.): Handbuch Digitale Geographien: Welt - Wissen - Werkzeuge. UTB, Stuttgart. (In Druck)

Resch, B. \& M. Szell (2019): Human-Centric Data Science for Urban Studies. In: ISPRS International Journal of Geo-Information 8(12). S. 584.

Roche, S. (2017): Geographic information science III: Spatial thinking, interfaces and algorithmic urban places Toward smart cities. In: Progress in Human Geography 41(5). S. 657-666.

Schulze, U., I. Gryl \& D. Kanwischer (2015): Spatial Citizenship: Zur Entwicklung eines Kompetenzstrukturmodells für eine fächerübergreifende Lehrerfortbildung. In: Zeitschrift für Geographiedidaktik 43(2). S. 139-164.
Seyfert, R \& J. Roberge (2017): Was sind Algorithmuskulturen? In: Seyfert, R. \& J. Roberge (Hrsg.). Algorithmuskulturen. Über die rechnerische Konstruktion der Wirklichkeit. Transcript-Verlag, Bielefeld. S. 7-40.

Spotify (2020): Amplifying Artist Input in Your Personalized Recommendations. https://newsroom.spotify. com/2020-11-02/amplifying-artist-input-in-your-personalized-recommendations/ (12.03.2021)

Stalder, F. (2016): Kultur der Digitalität. Suhrkamp, Berlin. Stalder, F. (2017): Algorithmen, die wir brauchen. https:// netzpolitik.org/2017/algorithmen-die-wir-brauchen (12.03.2021)

Swarat, G. (2018): Irgendwas mit Daten ...: Warum wir Data Scientists brauchen. In: Gesellschaft Für Informatik e.V. (Hrsg.): Data Literacy und Data Science Education: Digitale Kompetenzen in der Hochschulausbildung. Selbstverlag Gesellschaft für Informatik e.V., Berlin. S. $14-18$.

Unterberg, S. (2019): Drei von zehn Schülern können nur „Links anklicken und ihr Handy streicheln”. https:// www.spiegel.de/lebenundlernen/schule/computernutzung-ein-drittel-der-schueler-ist-abgehaengt-a-1294424. html (12.03.2021) 